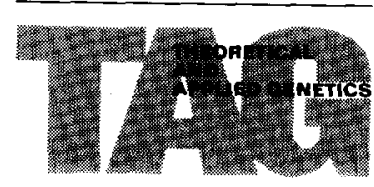

(C) Springer-Verlag 1987

\title{
Analysis of organelle genomes in a somatic hybrid derived from cytoplasmic male-sterile Brassica oleracea and atrazine-resistant $B$. campestris
}

\author{
D. Robertson $^{1, *}$, J. D. Palmer ${ }^{2}$, E. D. Earle ${ }^{1}$ and M. A. Mutschler ${ }^{1}$ \\ 1 Department of Plant Breeding, Cornell University, Ithaca. NY 14853-1902. USA \\ 2 Department of Biology, University of Michigan, Ann Arbor, MI 48109, USA
}

Received September 2, 1986; Accepted March 27, 1987

Communicated by P. Maliga

\begin{abstract}
Summary. An atrazine-resistant, male-fertile Brassica napus plant was synthesized by fusion of protoplasts from the diploid species B. oleracea and B. campestris. Leaf protoplasts from $B$. oleracea var. italica carrying the Ogura male-sterile cytoplasm derived from Raphanus sativus were fused with etiolated hypocotyl protoplasts of atrazine-resistant $B$. campestris. The selection procedure was based on the inability of $B$. campestris protoplasts to regenerate in the media used, and the reduction of light-induced growth of $B$. oleracea tissue by atrazine. A somatic hybrid plant that differed in morphology from both $B$. oleracea and $B$. campestris was regenerated on medium containing $50 \mu \mathrm{M}$ atrazine. Its chromosome number was 36-38, approximately that of B. napus. Furthermore, nuclear ribosomal DNA from this hybrid was a mixture of both parental rDNAs. Southern blot analyses of chloroplast DNA and an assay involving tetrazolium blue indicated that the hybrid contained atrazine-resistant $B$. campestris chloroplasts. The hybrid's mitochondrial genome was recombinant, containing fragments unique to each parent, as well as novel fragments carrying putative crossover points. Although the plant was female-sterile, it was successfully used to pollinate $B$. napus.
\end{abstract}

Key words: Somatic hybridization - Atrazine resistance - Mitochondrial recombinants - Brassica-Cytoplasmic male sterility

\section{Introduction}

Resynthesis of the amphidiploid species Brassica napus has been useful in plant breeding by increasing the

* Current address: Department of Zoology, Duke University, Durham, NC 27706, USA gene pool for cultivated $B$. napus varieties and has led to the development of new fodder or vegetable crops, a few of which have been released as varieties (Prakash and Hinata 1980). B. napus has been resynthesized from its progenitors, $B$. oleracea and $B$. campestris, using embryo and ovule culture but the success rate is low and the results are variable, depending in part upon the particular cultivars used (Namai et al. 1980). Protoplast fusion is an alternative method of resynthesis that was first successfully used by Schenck and Robbelen (1982) and more recently by Sundberg and Glimelius (1986) and Taguchi and Kameya (1986).

An advantage of protoplast fusion is that it can result in plants with novel combinations of organelleencoded traits. Cytoplasmically-encoded traits are usually inherited throug the maternal parent, and so it is difficult to separate chloroplast from mitochondriallyencoded traits. Fusion of somatic cells results in hybrid cytoplasms containing organelles from both parents; segregation during subsequent divisions of the hybrid cells can result in plants with new combinations of organelle-encoded traits, as well as recombinant organelle DNAs (reviewed by Fluhr 1983; Galun and Aviv 1983). In the genus Brassica, there are two cytoplasmically-encoded traits of agricultural interest, atrazine resistance and cytoplasmic male sterility (cms). Atrazine resistance, encoded on chloroplast DNA, has been transferred into the cultivated oil seed crops $B$. napus and $B$. campestris from an atrazine-resistant biotype of B. campestris (Beversdorf et al. 1980). The particular cms used in this study, the ogu cms (Ogura 1968), is associated with mitochondria (Pelletier et al. 1983) and has been crossed into $B$. campestris, $B$. oleracea, and B. napus.

Schenck and Robbelen (1982) relied on the poor regeneration ability of both $B$. campestris and $B$. 
oleracea compared to $B$. napus to select for somatic hybrids. We wondered whether the use of atrazine in vitro could also be used to reduce the number of atrazine-sensitive parental types. This report describes the synthesis of a male-fertile $B$. napus with atrazineresistant chloroplasts, produced by fusion of $\mathrm{cms} B$. oleracea (broccoli) and atrazine-resistant $B$. campestris.

\section{Materials and methods}

\section{Plant material}

Leaf and cotyledon protoplasts were isolated from Brassica oleracea var. italica cv Green Comet (GC) broccoli (Harris Moran Seed Co., Rochester, NY, USA). Most experiments used an Ogura $\mathrm{cms}$ line (GCcms) obtained by backcrossing an ogu $\mathrm{cms}$ broccoli with an inbred maintainer line derived from hybrid Green Comet. This line was developed by Dr. M. H. Dickson, New York Agricultural Experiment Station, Geneva, NY, USA. Etiolated hypocotyl protoplasts were isolated from $B$. campestris ssp. oleifera cv Candle, which contained atrazine-resistant chloroplasts. This line was developed by Dr. W. Beversdorf, University of Guelph, Guelph, Ontario, Canada. The rapid cycling strains of $B$. napus ( $\mathrm{CrGC12}$ ) and $B$. oleracea ( $\mathrm{CrGC}$ ) were obtained from the Crucifer Genetics Cooperative, Madison, WI, USA.

\section{Protoplast isolation and fusion}

Leaf and cotyledon protoplast donor plants were grown from seed in $500 \mathrm{ml}$ beakers with autoclaved vermiculite moistened with $1 / 2$ strength MS salts (Murashige and Skoog 1962), at $25^{\circ} \mathrm{C}$ with a $16 \mathrm{~h}$ photoperiod of $80 \mu \mathrm{E} / \mathrm{m}^{2} / \mathrm{s}$. Protoplasts were isolated from 3 young, fully expanded leaves of 24-day-old plants. Leaf tissue was scored with parallel cuts $1 \mathrm{~mm}$ apart and placed in enzyme diluted 1:8 with SCMP $(0.5 \mathrm{M}$ sorbitol, $10 \mathrm{mM} \mathrm{CaCl}_{2}, 5 \mathrm{mM}$ Mes, $1 \mathrm{mM} \mathrm{KH}_{2} \mathrm{PO}_{4}, \mathrm{pH} 5.8$ ). The enzyme stock solution consisted of $2 \% \mathrm{w} / \mathrm{v}$ Cellulase CELF, $0.1 \%$ Pectolyase Y-23, 0.5\% Driselase, $0.2 \mathrm{M}$ mannitol, $80 \mathrm{mM} \mathrm{CaCl}_{2}$, $1 \mathrm{mM} \mathrm{KH}_{2} \mathrm{PO}_{4}$ and $1 \mathrm{mM}$ Mes, $\mathrm{pH}$ 5.6. Etiolated hypocotyl protoplasts were isolated from 4-day-old $B$. campestris seedlings grown in the dark at $25^{\circ} \mathrm{C}$ on medium A (Pelletier et al. 1983). About 30 hypocotyls were slit longitudinally and placed in enzyme diluted 1:4 with SCMP. After $16 \mathrm{~h}$ incubation at $50 \mathrm{rpm}$ at $30^{\circ} \mathrm{C}$, leaf protoplasts were diluted with SCMP and hypocotyl protplasts with $0.18 \mathrm{M} \mathrm{CaCl}_{2}$ and $1 \mathrm{mM} \mathrm{Mes,} \mathrm{pH} 5.8$. Protoplasts were centrifuged $5 \mathrm{~min}$ at $55 \mathrm{~g}$ (leaf) or $75 \mathrm{~g}$ (hypocotyl). Protoplast pellets were resuspended in $3 \mathrm{ml} 0.5 \mathrm{M}$ sucrose with $1 \mathrm{mM}$ Mes ( $\mathrm{pH} 5.8$ ), a small amount of SCMP was layered on top and the tubes were centrifuged $5 \mathrm{~min}$ at $55 \mathrm{~g}$. The floating bands from both tubes were combined and diluted with SCMP to a final concentration of 2.5 to $5 \times 10^{5} / \mathrm{ml}$. The ratio of GCcms to $B$. campestris protoplasts was $1: 1.3$.

The procedure for fusion was derived from that of Douglas et al. (1981). Polyethylene glycol (PEG) 6,000 was prepared as a $33 \% \mathrm{w} / \mathrm{v}$ solution in $0.2 \mathrm{M}$ glucose, $10 \mathrm{mM} \mathrm{CaCl}_{2}$ and $0.7 \mathrm{mM} \mathrm{KH}_{2} \mathrm{PO}_{4}$ and filter sterilized. One milliliter of the protoplast mixture was placed in each of four $15 \mathrm{ml}$ conical test tubes and $1 \mathrm{ml}$ of the PEG solution was gradually added down the side of each tube and gently mixed. After $15 \mathrm{~min}$, $0.5 \mathrm{ml} \mathrm{SCMP}$ was added and mixed. At intervals of $5 \mathrm{~min}$, $1 \mathrm{ml}, 2 \mathrm{ml}, 4 \mathrm{ml}$, and $4 \mathrm{ml}$ of $0.18 \mathrm{M} \mathrm{CaCl}_{2}$ were added and gently mixed. Tubes were centrifuged $5 \mathrm{~min}$ at $75 \mathrm{~g}$ and the pellets resuspended in $4 \mathrm{ml} \mathrm{SCMP}$. After centrifugation protoplasts were washed once in medium B (Pelletier et al. 1983).
They were plated in $0.5 \mathrm{ml}$ aliquots of medium B in 24-well multiwell plates (Falcon) at a concentration of $5 \times 10^{4} / \mathrm{ml}$.

\section{Plant regeneration}

The procedures described by Robertson and Earle (1986) were followed, using the series of media (B-G) developed by Pelletier et al. (1983). Protoplasts were kept in the dark for 2 days and then exposed to $80 \mu \mathrm{E} / \mathrm{m}^{2} / \mathrm{s}$ light (provided by a combination of Gro-lux and cool white fluorescent lights) in a $16 \mathrm{~h}$ photoperiod at $25^{\circ} \mathrm{C}$. After 4 weeks of culture, protoplast-derived calli ( $\mathrm{p}$-calli) were transferred to medium $\mathrm{E}$ solidified with $0.22 \%$ Gelrite with or without different concentrations of atrazine. A $50 \mathrm{mM}$ stock solution of atrazine (gift of Ciba Geigy) was prepared in $95 \%$ ethanol, stored at $-20^{\circ} \mathrm{C}$, and added to autoclaved medium E. Shoots initiated on medium $E$ were transferred to Gelrite-solidified media $F$ and $\mathrm{G}$ without atrazine. Plants that did not root on medium $\mathrm{G}$ were rooted in medium containing $1 \%$ sucrose, $1 / 2$ strength MS salts, $0.1 \mathrm{mg} / \mathrm{l} \mathrm{NAA}$ and $1 \%$ agar in Magenta GA-7 boxes. All plants were hardened as described by Robertson and Earle (1986). Chromosome numbers of regenerated plants were determined from dividing microsporocytes stained with acetocarmine.

\section{Determination of atrazine resistance}

The tetrazolium blue assay (Robertson and Earle 1987) was used to determine atrazine resistance. Protoplasts were suspended in medium containing $0.6 \mathrm{M}$ sorbitol, $50 \mathrm{mM}$ Hepes (pH 7.6) and $10 \mathrm{mM} \mathrm{NaHCO}_{3}$ with or without $50 \mu \mathrm{M}$ atrazine. Nitro-blue tetrazolium (Sigma) was added to a concentration of $0.01 \%$ and the protoplasts were exposed to $150 \mu \mathrm{E} / \mathrm{m}^{2} / \mathrm{s}$ light for $30 \mathrm{~min}$. The degree of staining of 500 protoplasts was evaluated using an inverted microscope.

\section{Molecular characterization}

Total cellular DNA was prepared from the atrazine-resistant somatic hybrid and its fusion parents by the procedure of Saghai-Maroof et al. (1984). The DNAs were further purified by two bandings in $\mathrm{CsCl}$ /ethidium bromide and dialysis. Restriction endonuclease digestions, agarose gel electrophoresis, bidirectional transfers of DNA fragments from agarose gels to Zetabind (AMF Cuono) hybridization membranes, labeling of recombinant plasmids by nick-translation, and filter hybridizations were performed as described (Palmer 1982, 1985).

\section{Results}

\section{Protoplast fusion and regeneration of hybrid}

The fusion procedure consistently produced $1 \%-6 \%$ heterokaryotic cells with transvacuolar strands from the $B$. campestris hypocotyl protoplasts and chloroplasts from the $B$. oleracea leaf protoplasts. In the experiment reported here, 1,089 p-calli showed growth on solidified medium $\mathrm{E} 4$ weeks after the fusion procedure. After 5 weeks, equal numbers of these calli, as well as about 200 calli from control GCcms protoplasts not exposed to PEG, were transferred to medium $E$ with or without $50 \mu \mathrm{M}$ atrazine. Although unfused $\mathrm{GCcms}$ control protoplasts from this experiment developed to the callus stage, no shoots were regenerated on medium $\mathrm{E}$ with or 


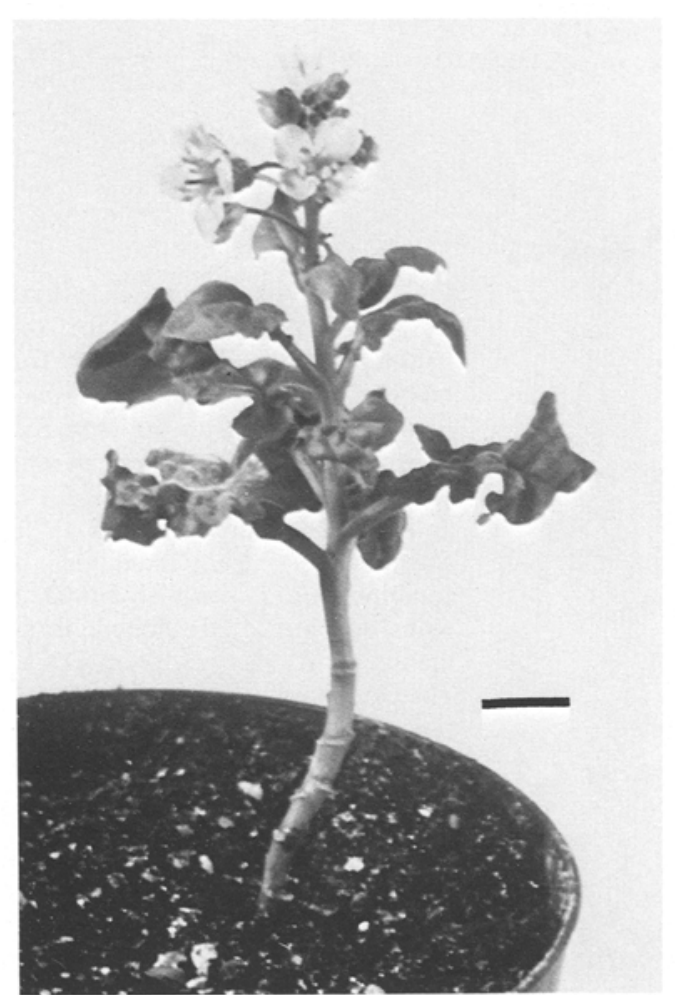

Fig. 1. Morphology of the somatic hybrid at the flowering stage. $\mathrm{Bar}=1 \mathrm{~cm}$

without atrazine. In previous studies (Robertson and Earle 1986), GCcms also had a low morphogenetic potential. The frequency of shoot formation of the 1,089 calli from the fusion treatment was low but 4 shoots were recovered. Three were regenerated on medium lacking atrazine. They were vitreous, lacked trichomes, and were not successfully hardened.

The fourth shoot was regenerated on medium containing $50 \mu \mathrm{M}$ atrazine. Trichomes were visible on this shoot when it was $2 \mathrm{~cm}$ high. As $B$. campestris, which has trichomes, does not regenerate from protoplasts on the media used and as broccoli lacks trichomes, the plantlet was tentatively identified as a somatic hybrid. The shoot did not root in medium $\mathrm{G}$ (without atrazine) but root formation did occur on medium containing $1 / 2$ MS salts, $1 \%$ sucrose, $1 \%$ agar and $0.1 \mathrm{mg} / 1$ NAA. Over 30 clones derived from axillary buds of this hybrid were propagated by placing excised nodes on rooting medium. Even successfully hardened clones were difficult to grow because root development was poor. The first clone flowered one year after the fusion experiment, shortly after transfer from a $16 \mathrm{~h}$ photoperiod at $20^{\circ} \mathrm{C}$ to continuous light of comparable intensity at $25^{\circ} \mathrm{C}$.

The morphology of the somatic hybrid (Fig. 1) differed from both parents. The leaves were rugose, and

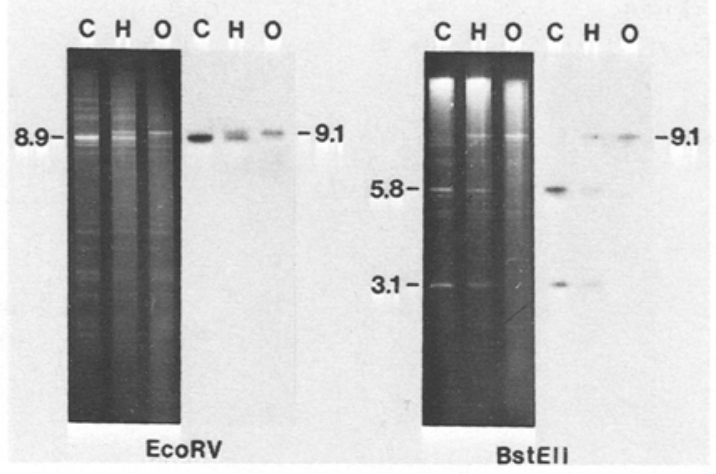

Fig. 2. Analysis of nuclear rDNA sequences in the atrazine-resistant somatic hybrid and its parents. Total cellular DNAs from the $B$. campestris parent $(C)$, the hybrid $(H)$, and the $B$. oleracea parent $(O)$ were digested with EcoRV and BstEII and electrophoresed on a 1.0\% agarose gel (left half of each panel). The DNA fragments were transferred to a zetabind filter and hybridized with nick-translated pHAl (right half of each panel). Fragment sizes are given in $\mathbf{k b}$

Table 1. Reduction of nitro-blue tetrazolium in protoplasts from atrazine-susceptible and -resistant lines of $B$. napus $\mathrm{cv}$ Tower and from the somatic hybrid

\begin{tabular}{lcc}
\hline Protoplast source & $\begin{array}{c}\text { Atrazine } \\
0\end{array}$ & $\begin{array}{l}(\mu M) \\
50\end{array}$ \\
\hline B. napus, atrazine-susceptible & $80 \%^{\circ}$ & $0 \%$ \\
B. napus, atrazine-resistant & $92 \%$ & $86 \%$ \\
somatic hybrid & $86 \%$ & $84 \%$ \\
\hline
\end{tabular}

a Percentage of stained protoplasts in 500 protoplasts counted

the stem was thicker than B. campestris. Young leaves and stems were densely covered with trichomes. As the plant matured, the density of trichomes decreased, and at flowering it completely lacked trichomes and had a waxy bloom. The plant was $10 \mathrm{~cm}$ tall when it flowered. Its chromosome number was $36-38$, which is close to the diploid chromosome number for $B$. napus $(2 \mathrm{n}=38)$.

Analysis of nuclear ribosomal DNA sequences confirmed that the putative somatic hybrid does indeed possess a hybrid nucleus. Plasmid pHAl, which contains a monomer length fragment of nuclear ribosomal DNA from pea (Jorgensen et al. 1982), was hybridized to Southern blots containing total DNA from the hybrid and its parents, each digested with four enzymes (Xho I, BstE II, EcoR V, Xba I) known (J. D. Palmer, unpublished data) to distinguish the parental nuclear rDNAs. As expected, the hybrid contained the full complement of both parental sets of bands with each of the four enzymes, results for two of which are shown in Fig. 2. 


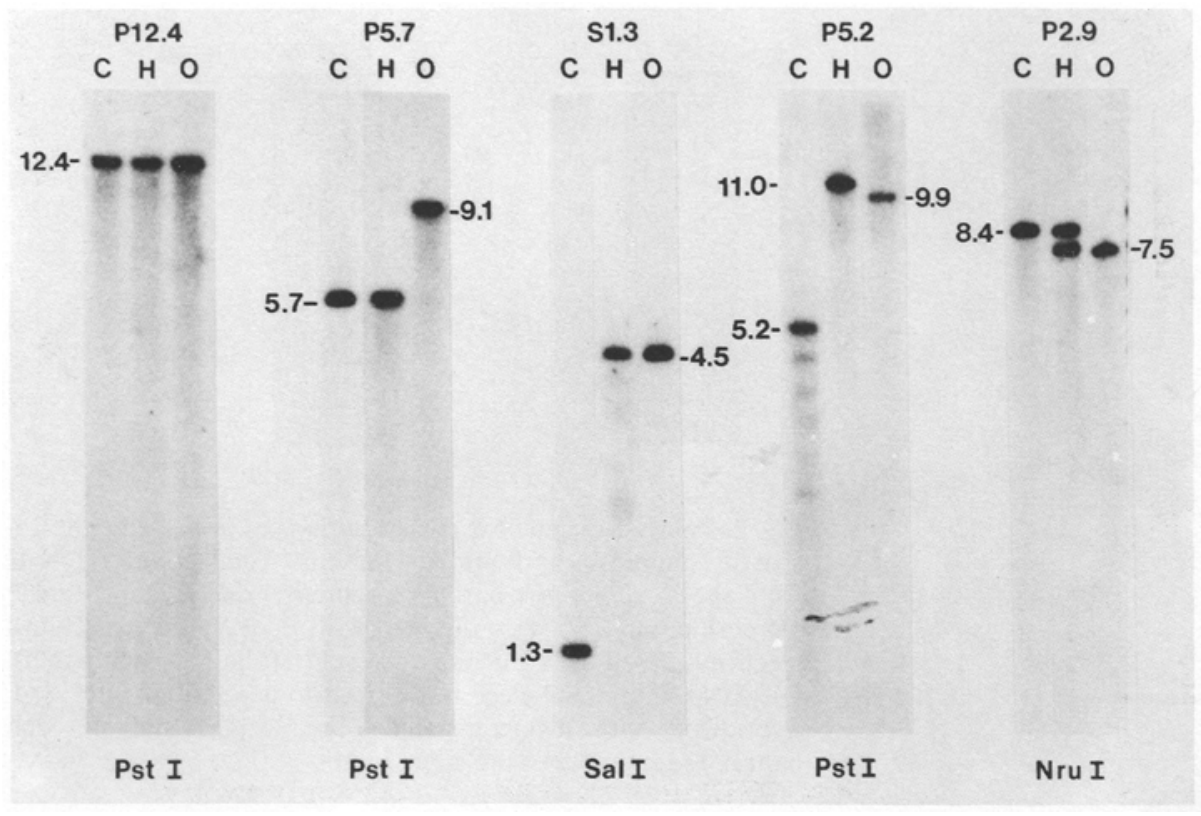

Fig. 3. Analysis of mitochondrial DNA sequences in the atrazine-resistant hybrid and its parents. Total cellular DNAs from the B. campestris parent $(C)$, the somatic hybrid $(H)$, and $B$. oleracea parent $(O)$ were digested with Pst I, Sal I, and Nru I, separated on a $0.7 \%$ agarose gel, transferred to a zetabind filter, and hybridized with the indicated cloned fragments of $B$. campestris mtDNA. The clone designations indicate the type of fragment cloned ("P" for Pst I and "S" for Sal I) and its size in $\mathrm{kb}$
The somatic hybrid was male-fertile, with a flower morphology similar to B. campestris. Although pollen from this plant was successfully used in crosses with rapid cycling $B$. napus ( $\mathrm{CrGCl}$ ), the plant was femalesterile, even when buds or open flowers were selfpollinated or pollinated from $\mathrm{CrGC} 12$ or $\mathrm{CrGC} 3$ (rapid cycling B. oleracea).

\section{Analysis of organellar traits}

The tetrazolium blue assay was used to determine whether the regenerated plants contained atrazineresistant chloroplasts. In this assay, reduction of nitroblue tetrazolium by photosystem II electron transport leads to visible staining of chloroplasts within protoplasts. Protoplasts from atrazine-resistant and atrazinesensitive $B$. napus show comparable staining in the absence of atrazine; addition of atrazine inhibits staining of the sensitive but not the resistant protoplasts (Table 1). There was no reduction in staining of protoplasts from the somatic hybrid in medium containing $50 \mu \mathrm{M}$ atrazine (Table 1), indicating that this plant was atrazine-resistant.

Based on its resistance to atrazine, a cpDNAencoded trait carried by the $B$. campestris parent, we expected the hybrid to contain the $B$. campestris chloroplast genome. To confirm this expectation, total DNA from the hybrid and its parents was digested with each of four enzymes (Bgl I, Sac II, Sal I, Sma I) known (Palmer et al. 1983) to distinguish the parental cpDNAs and hybridized with appropriate cloned cpDNA fragments. With every enzyme tested the hybrid was found to contain a $B$. campestris-specific cpDNA restriction fragment or fragments (hybridizations not shown). The five restriction site mutations that distinguish the parental genomes with the four enzymes used are scattered widely in the large single copy region and inverted repeat of the Brassica chloroplast genome (Palmer et al. 1983). Therefore, it seems likely that the hybrid contains exclusively $B$. campestris cpDNA, although we connot rule out the possibility that the hybrid chloroplast genome contains relatively small portions of the Ogura chloroplast genome (which would have to be indistinguishable from the homologous portions of the $B$. campestris genome with the enzymes used) as the result of recombination between parental cpDNAs.

In contrast to the straightforward compostion of the hybrid's nuclear and cloroplast genomes, its mitochondrial genome was found to consist of a complex mixture of the parental mtDNAs. Figure 3 shows the hybridization patterns of five representative $B$. campestris mtDNA clones (Palmer and Shields 1984) to total DNAs from the hybrid and its parents. P12.4, a clone containing a $12.4 \mathrm{~kb}$ Pst I fragment, hybridized to identically-sized fragments in the two parents and in the hybrid. In contrast, each of the other four clones hybridized to a single species-specific band in each of the parental DNAs. Moreover, each of these clones revealed a different pattern of fragments for the hybrid relative to its parents. P5.7 hybridized to a single fragment in the hybrid of the same size as in the $B$. campestris parent, while, conversely, $\mathrm{S} 1.3$ hybridized to a single $B$. oleracea-specific fragment in the hybrid (Fig. 3). Thus, it appears that the mitochondrial ge- 
nome of the hybrid is recombinant, containing specific portions of each of the parental genomes.

More complex results, also consistent with the recombination hypotheses, were observed with the other two clones used in Fig. 3. P5.2 hybridized to a single fragment in the hybrid; this fragment differs in size from either of the parental-specific fragments. We interpret this hybrid fragment as containing a crossover point for recombination between the parental genomes, i.e., this fragment should contain a portion of each parental genome. P2.9 hybridized to two fragments in the hybrid, one corresponding in size to the $B$. campestris-specific fragment and the other to the $B$. oleraceaspecific fragment (Fig. 3). Thus, the hybrid appears to contain both parental sequences from this region of the genome, so that sequences present only once in either parent are duplicated in the hybrid. This result is consistent with simple models of recombination between parental genomes if we assume that the $8.4 \mathrm{~kb}$ and $7.5 \mathrm{~kb}$ parental homologous sequences are located on opposite sides of the recombining chromosomes relative to a point of reciprocal crossover. Such a topology is easily imagined, given that the mitochondrial chromosomes from the parents are known to be highly rearranged as the result of a dozen or so large inversions (J. D. Palmer and L. A. Herbon, unpublished results).

\section{Discussion}

One goal of this work was to develop improved methods for obtaining Brassica somatic hybrids. Although the frequency of shoot formation was low (less than $1 \%$ of the calli produced shoots) one of them was a somatic hybrid. Another goal, to begin study of the genetics of cms, was accomplished in that the malefertile somatic hybrid contained a $\mathrm{mt}$ genome recombinant between male fertility- and male sterility-encoding $\mathrm{mt}$.

Our previous experiments (Robertson and Earle 1986) indicated that $\mathrm{GCcms}$ P-calli formed shoots less frequently than p-calli from fertile, hybrid GC. In the experiment reported here, about 200 calli from unfused control GCcms protoplasts formed no shoots on medium $\mathrm{E}$ and protoplasts from unfused $B$. campestris divided but did not develop to a point where they could be transferred to agar. Other investigators have also noted that $B$. campestris does not regenerate easily from protoplasts (Xu et al. 1982; Lu et al. 1982; Glimelius 1984). Only $0.7 \%$ of calli from protoplasts treated with PEG (3/545) formed shoots on medium $E$ without atrazine. The low totipotency of both parental species may in part explain the low frequency of regeneration in the fusion experiment and may also have contributed to the selection of the B. napus-like fusion product.

Only one plant regenerated on medium containing atrazine; this plant was atrazine-resistant, male fertile, and had a diploid chromosome number similar to that of $B$. napus. Pelletier et al. (1983) used B. napus, which shows high frequency regeneration from protoplasts, as a source of atrazine resistance. Protoplasts from atrazine resistant $B$. napus and $\mathrm{cms} B$. napus were fused but only 2 out of 85 regenerated plants contained atrazine resistant chloroplasts.

Experiments using 4-week-old p-calli from fertile, hybrid Green Comet showed that $1-50 \mu \mathrm{M}$ atrazine in medium $\mathrm{E}$ with $1 \%$ sucrose reduced the growth rate in the light by a factor of 4 , to a rate comparable to darkgrown calli (data not shown). Grant et al. (1983) also reported that atrazine reduced the growth of atrazine sensitive $B$. napus callus to levels of dark-grown callus and that growth of atrazine-resistant $B$. napus callus was not inhibited by atrazine. As the number of shoots regenerated in the fusion experiment reported here was small (four), it was not possible to determine the effectiveness of atrazine as an in vitro selective agent.

Schenck and Robbelen (1982) fused protoplasts from 1.236 different combinations of varieties and cultivars of $B$. oleracea and $B$. campestris but were able to regenerate only 3 true synthetic $B$. napus plants. The experiments reported here used only two lines, derived from the cultivars Green Comet and Candle, and resulted in the regeneration of one synthetic $B$. napus. Taguchi and Kameya (1986) also regenerated two synthetic $B$. napus derived from fusion of leaf protoplasts from two lines: Chinese cabbage (B. campestris) and cabbage ( $B$. oleracea). Although they found that calli derived from fusion products showed more vigorous development than calli derived from unfused protoplasts, we were unable to detect a difference between development of calli from unfused GCcms protoplasts and the protoplast fusion products. We used different media for protoplast development, and so our results are not strictly comparable. Sundberg and Glimelius (1986) have also regenerated B. napus from fusion of protoplasts from $B$. oleracea and $B$. campestris. Using micromanipulation they were able to select fusion products at an early stage and obtained 450 hybrid calli and about $1 \%$ shoot formation. This low frequency of shoot formation emphasizes the need for good selection techniques.

The rDNA analysis indicated that at the time of analysis genome components from both parents were present in approximately equal amounts in the somatic hybrid. This technique, which has also been used in tobacco (Uchimaya et al. 1983), was especially useful in determining the nuclear constitution of the somatic hybrid since poor root development made root tip 
chromosome counts difficult and the plant took 1 year to flower. The estimated chromosome numer of the somatic hybrid was 36-38. The rDNA analysis eliminated the possibility that the somatic hybrid was a tetraploid $B$. campestris $(4 n=40)$ rather than a diploid B. napus $(2 \mathrm{n}=38)$.

The hybrid regenerated in this study was self- and female-sterile, although it was successfully used to pollinate rapid-cycling $B$. napus. The three synthetic amphidiploids regenerated by Schenck and Robbelen (1982) were self-fertile although the number of seed produced was small.

The tetrazolium blue assay provided a convenient method for determining atrazine resistance. Since it requires only a small amount of tissue, it could be performed as soon as the leaves from the hybrid showed normal (non-vitreous) morphology. Results from Southern hybridizations confirmed those of the tetrazolium blue assay by showing that the atrazineresistant somatic hybrid contained $B$. campestris chloroplast DNA. Although Medgyesy et al. (1985) reported recombination of chloroplast DNA in an interspecific Nicotiana somatic hybrid, in all other somatic hybrids chloroplast DNA recombination has not been detected (Fluhr et al. 1983).

The hybrid's mitochondrial genome contained restriction fragments specific to each parent, as well as novel fragments unlike those of either parent. Although the latter observation alone could be explained by internal rearrangement of mtDNA, as sometimes occurs during tissue culture (Gengenbach et al. 1981; Kemble et al. 1982; Kemble and Shepard 1984), the two observations together strongly suggest recombination between parental mtDNA, leading to the creation of a "hybrid" mitochondrial genome. Evidence for mtDNA recombination in somatic hybrids has also been described in tobacco (Belliard et al. 1979; Nagy et al. 1983; Galun et al. 1982), Petunia (Boeshore et al. 1983), Brassica (Chetrit et al. 1985) and Daucus (Matthews and Widholm 1985). The strongest evidence in favor of the recombination hypothesis is that of Rothenberg et al. (1985), who cloned a novel mtDNA fragment from a Petunia somatic hybrid and showed that it contained restriction sites from both parents.

Our current studies are taking advantage of the relatively small and well-understood mitochondrial genome of Brassica species (Palmer and Shields 1984) in an attempt to determine the complete structure of the recombinant $\mathrm{mt}$ genome in this hybrid and to identify the exact points of crossover. Furthermore, by analyzing the mitochondrial genomes of additional hybrids, both male-fertile and sterile, we hope to identify specific portion(s) of the genome that may be involved in sterility, as Boeshore et al. (1985) were able to do in Petunia.
Acknowledgements. We thank L. Herbon and J. Nugent for assistance with the molecular experiments, and $\mathrm{R}$. Cuellar for providing plasmid $\mathrm{pHAl}$. The assistance of E. Cobb and O. S. Jourdan in pollinations and fertility assessment is gratefully acknowledged. This research was supported by USDA grant 85-00363 and NSF grant DCB-8207701 to M.A. Mutschler and E.D. Earle, and by NIH grant GM-32680 to J.D. Palmer.

\section{References}

Belliard G, Vedel F, Pelletier G (1979) Mitochondrial recombination in cytoplasmic hybrids of Nicotiana tabacum by protoplast fusion. Nature (London) 281:401-403

Beversdorf WD, Weiss-Lerman J, Erickson LR, SouzaMachado V (1980) Transfer of cytoplasmically-inherited triazine resistance from birds-rape to cultivated oil seed rape (B. campestris and B. napus). Can J Genet Cytol 22: $167-172$

Boeshore ML, Lifshitz I, Hanson MR, Izhar S (1983) Novel composition of mitochondrial genomes in Petunia somatic hybrids derived from cytoplasmic male sterile and fertile plants. Mol Gen Genet 190:459-467

Boeshore ML, Hanson MR, Izhar S (1985) A variant mitochondrial DNA arrangement specific to Petunia stable sterile somatic hybrids. Plant Mol Biol 4:125-132

Chetrit P, Mathieu C, Vedel F, Pelletier G, Primard C (1985) Mitochondrial DNA polymorphism induced by protoplast fusion in Cruciferae. Theor Appl Genet 69:361-366

Douglas GC, Keller WA, Setterfield G (1981) Somatic hybridization between Nicotiana rustica and $N$. tabacum. II. Protoplast fusion and selection and regeneration of hybrid plants. Can J Bot 59:220-227

Fluhr R (1983) The segregation of organelles and cytoplasmic traits in higher plant somatic fusion hybrids. In: Potrykus I, Harms CT, Hinnen A, Hütter R, King PS, Shillito RD (eds) 6th Int Protoplast Symp Proc. Birkhäuser, Basel, pp 85-92 (Exp Suppl 46)

Fluhr R, Aviv D, Edelman M, Galun E (1983) Cybrids containing mixed and sorted-out chloroplasts following interspecific somatic fusions in Nicotiana. Theor Appl Genet 65:289-294

Galun E, Aviv D (1983) Cytoplasmic hybridization: genetic and breeding applications. In: Evans DA, Sharp WR, Ammirato PV, Yamada Y (eds) Techniques for propagation and breeding. Handbook of plant cell culture, vol 1. Macmillan, New York, pp 358-389

Galun E, Arzee-Gonen P, Fluhr R, Edelman M, Aviv D (1982) Cytoplasmic hybridization in Nicotiana: mitochondrial DNA analysis in progenies resulting from fusion between protoplasts having different organelle constitutions. Mol Gen Genet 186: 150-156

Gengenbach BG, Connelly JA, Pring DR, Conde MF (1981) Mitochondrial DNA variation in maize plants regenerated during tissue culture selection. Theor Appl Genet 59: $161-167$

Glimelius K (1984) High growth rates and regeneration capacity of hypocotyl protoplasts in some Brassicaceae. Physiol Plant 61:38-44

Grant I, Beversdorf WD, Zilka JH (1983) Response of lightand darkgrown callus of atrazine-resistant and susceptible rapeseed (Brassica napus) to varying concentrations of atrazine. Plant Cell Tiss Org Cult 2:185-189

Jorgensen RA, Cuellar RE, Thompson WF (1982) Modes and tempos in the evolution of ribosomal RNA genes in legumes. Carnegie Inst Yearbook 81:98-101 
Kemble RJ, Shepard, JF (1984) Cytoplasmic DNA variation in a potato protoclonal population. Theor Appl Genet 69 211-216

Kemble RJ, Flavell RB, Brettell RIS (1982) Mitochondrial DNA analyses of fertile and sterile maize plants derived from tissue culture with the Texas male sterile cytoplasm. Theor Appl Genet 62:213-217

Lu DY, Pental D, Cocking EC (1982) Plant regeneration from seedling cotyledon protoplasts. Z Pflanzenphysiol 107: $59-63$

Matthews BF, Widholm JM (1985) Organelle DNA compositions and isoenzyme expression in an interspecific somatic hybrid of Daucus. Mol Gen Genet 198:371-376

Medgyesy P, Fejes E, Maliga P (1985) Interspecific chloroplast recombination in a Nicotiana somatic hybrid. Proc Natl Acad Sci USA 82:6960-6964

Murashige T, Skoog F (1962). A revised medium for rapid growth and bioassays with tobacco tissue cultures. Physiol Plant 15:473-497

Nagy F, Lazar G, Menczel L, Maliga P (1983) A heteroplasmic state induced by protoplast fusion is a necessary condition for detecting rearrangements in Nicotiana mitochondrial DNA. Theor Appl Genet 66:203-207

Namai H, Sarashima M, Hosoda T (1980) Interspecific and intergeneric hybridization breeding in Japan. In: Tsunoda S, Hinata K, Gomez-Campo C (eds) Brassica crops and wild allies, biology and breeding. Jpn Sci Soc Press, Tokyo, pp 191-203

Ogura H (1968) Studies on the new male-sterility in Japanese radish, with special reference to the utilization of this sterility towards practical raising of hybrid seed. Mem Fac Agric Kagoshima Univ 6:39-78

Palmer JD (1982) Physical and gene mapping of chloroplast DNA from Atriplex triangularis and Cucumis sativa. Nucleic Acids Res 10: 1593-1605

Palmer JD (1985) Isolation and structural analysis of chloroplast DNA. Methods Enzymol 118: 167-186

Palmer JD, Shields CR (1984) Tripartite structure of Brassica campestris mitochondrial genome. Nature (London) 307: $437-440$
Palmer JD, Shields CR, Cohen DB, Orton TJ (1983) Chloroplast DNA evolution and the origin of amphidiploid Brassica species. Theor Appl Genet 65:181-189

Pelletier G, Primard C, Vedel F, Chetrit P, Remy R, Rouselle P, Renard M (1983) Intergeneric cytoplasmic hybridization in Cruciferae by protoplast fusion. Mol Gen Genet 191: 244-252

Prakash S, Hinata K (1980) Taxonomy, cytogenetics and origin of crop Brassicas, a review. Opera Bot 55:1-57

Robertson D, Earle ED (1986) Plant regeneration from leaf protoplasts of Brassica oleracea var italica cv Green Comet broccoli. Plant Cell Rep 5:61-64

Robertson D, Earle ED (1987) Nitro-blue tetrazolium: a stain for photosynthesis in protoplasts. Plant Cell Rep 6:70-73

Rothenberg M, Boeshore ML, Hanson MR, Izhar S (1985) Intergenomic recombination of mitochondrial genomes in a somatic hybrid plant. Curr Genet 9:615-618

Saghai-Maroof MA, Soliman KM, Jorgensen RA, Allard RS (1984) Ribosomal DNA spacer-length polymorphisms in barley: Mendelian inheritance, chromosomal location, and population dynamics. Proc Natl Acad Sci USA 81: 8014-8018

Schenck HR, Robbelen G (1982) Somatic hybrids by fusion of protoplasts from Brassica oleracea and $B$. campestris. Z Pflanzenzücht 89:278-288

Sundberg E, Glimelius K (1986) A method for production of interspecific hybrids within Brassiceae via somatic hybridization, using resynthesis of Brassica napus as a model. Plant Sci 43: 155-162

Taguchi T, Kameya T (1986) Production of somatic hybrid plants between cabbage and Chinese cabbage through protoplast fusion. Jpn J Breed 36:185-189

Uchimaya H, Ohgawara T, Kato $H$, Akiyama T, Harada H (1983) Detection of two different nuclear genomes in parasexual hybrids by ribosomal RNA gene analysis. Theor Appl Genet 64: 117-118

Xu Z-H, Davey MR, Cocking EC (1982) Plant regeneration from root protoplasts of Brassica. Plant Sci Lett 24: $117-121$ 